\title{
Maximizing Networking Capacity in Multi-Channel Multi-Radio Wireless Networks
}

\author{
Pengjun $\mathrm{Wan}^{1}$ (万鹏俊) and Zhi-Guo $\mathrm{Wan}^{2}$ (万志国) \\ ${ }^{1}$ Department of Computer Science, Illinois Institute of Technology, Chicago, IL 60616, U.S.A. \\ ${ }^{2}$ School of Software, Tsinghua University, Beijing 100084, China \\ E-mail: wan@cs.iit.edu; wanzhiguo@tsinghua.edu.cn
}

Received March 11, 2014; revised June 30, 2014.

\begin{abstract}
Providing each node with one or more multi-channel radios offers a promising avenue for enhancing the network capacity by simultaneously exploiting multiple non-overlapping channels through different radio interfaces and mitigating interferences through proper channel assignment. However, it is quite challenging to effectively utilize multiple channels and/or multiple radios to maximize throughput capacity. The National Natural Science Foundation of China (NSFC) Project 61128005 conducted comprehensive algorithmic-theoretic and queuing-theoretic studies of maximizing wireless networking capacity in multi-channel multi-radio (MC-MR) wireless networks under the protocol interference model and fundamentally advanced the state of the art. In addition, under the notoriously hard physical interference model, this project has taken initial algorithmic studies on maximizing the network capacity, with or without power control. We expect the new techniques and tools developed in this project will have wide applications in capacity planning, resource allocation and sharing, and protocol design for wireless networks, and will serve as the basis for future algorithm developments in wireless networks with advanced features, such as multi-input multi-output (MIMO) wireless networks.
\end{abstract}

Keywords multi-channel multi-radio, wireless interference, network capacity, approximation algorithm, stability analyses

\section{Introduction}

Providing each node with one or more multi-channel radios offers a promising avenue for enhancing the network capacity by simultaneously exploiting multiple non-overlapping channels through different radio interfaces and mitigating interferences through proper channel assignment. However, it is quite challenging to effectively utilize multiple channels and/or multiple radios to maximize throughput capacity. The throughput maximization in multi-channel multi-radio (MC-MR) multihop wireless networks involves four correlated subproblems in the top-down order. First, for each end-toend communication request, we need to find a group of routing paths and compute the amount of traffic carried over each of these paths. Second, for each communication link, we have to properly distribute its total traffic demand to all possible radio-links joining the two end nodes of this communication links. Third, for each radio-link, we need to further split its traffic demand to be transmitted over individual channels. Forth, for each radio-link over each channel, we must assign a sequence of transmitting time-intervals subject to the constraint that, at any time, all scheduled links can transmit successfully. Therefore, a joint solution should be developed for routing, radio assignment, channel assignment, and transmission scheduling altogether. The primary objective of the National Natural Science Foundation of China (NSFC) Project 61128005 is to develop efficient and effective algorithmic solutions to maximize the network capacity of MC-MR wireless networks.

During the two-year period of this project, we conducted comprehensive algorithmic-theoretic and queuingtheoretic studies of maximizing wireless networking capacity in MC-MR wireless networks under the protocol interference model and fundamentally advanced the state of the art from three aspects.

1) We have developed efficient approximation algorithms with provably good performance guarantees for various capacity optimization problems. The prevailing paradigm of existing algorithmic studies of multiflow problems in wireless networks resorted to the traditional linear programming methods, which is quite inefficient in both running time and memory requirement. For the first time ever, we shifted to a new purely combinatorial paradigm which involves only a sequence of shortest-path computations but still achieves provably

Regular Paper

This work was supported in part by the National Natural Science Foundation of China under Grant No. 61128005.

C) 2014 Springer Science + Business Media, LLC \& Science Press, China 
good approximation factors. Thus, the algorithmic solutions developed in this project are not only faster but also simpler and easier for distributed implementation. Furthermore, they offer a quantized trade-off between accuracy and efficiency.

2) We also developed a number of greedy wireless link scheduling algorithms with provably good stable efficiency. For the first time, we also derived stability subregions with explicit closed forms which are shown to be within a constant factor of the network capacity region, which has been missing even in singlechannel single-radio (SC-SR) wireless networks. The polynomial-time membership testability of these stability subregions is particularly favorable for cross-layer optimization, where one needs to allocate the link rates efficiently while still ensuring the network stability. Our analyses were accomplished by an innovative samplepath argument, which pushes the deterministic arguments as far as possible while trying to avoid the heavy machinery of stochastic processes.

3) The quantified interference factor and interference-aware link congestions and lengths introduced in this project essentially capture the impact of wireless interference. The deep relation between interferenceaware shortest paths and the maximum (concurrent) multiflow is a cornerstone to combinatorial algorithms for all multiflow problems in wireless networks. Furthermore, the new techniques and tools developed in this project will have wide applications in capacity planning, resource allocation and sharing, and protocol design for wireless networks, and will serve as the basis for future algorithm developments in wireless networks with advanced features, such as multi-input multi-output (MIMO) wireless networks.

In addition, we have taken initial algorithmic studies on maximizing the network capacity under the physical interference model.

This article is a survey on our major discoveries made in the NSFC Project 61128005. The purpose of this article is to highlight the new concepts and methodologies developed in this project, while skipping the technical details which can be found in the references. Section 2 introduces the network model and various optimization problems involved in maximizing the wireless network capacity. Section 3 and Section 4 are devoted to algorithmic studies of the maximization of the network capacity under the protocol interference model and the physical interference model respectively. Section 5 analyzes the stable efficiencies as well as the stable capacity subregions of several greedy link scheduling algorithms. Finally, we conclude this article in Section 6.

\section{Network Model and Problem Descriptions}

Throughout this article, the MC-MR wireless network consists of $\lambda$ non-overlapping channels, a set $V$ of $n$ wireless nodes, and $A$ is the set $V$ of $m$ direct nodelevel communication links. Each node $v$ has $\tau(v)$ radios. Let $\mathcal{I}$ denote the collection of all independent subsets of $A$ specified by the adopted interference model. For each node-level link $a=(u, v)$ in $A$, we make $\lambda \cdot \tau(u) \cdot \tau(v)$ replications $(u, v, i, j, l)$ for $1 \leqslant i \leqslant \tau(u), 1 \leqslant j \leqslant \tau(v)$, and $1 \leqslant l \leqslant \lambda$. A replication $(u, v, i, j, k)$ always utilizes the $i$-th radio at $u$ and the $j$-th radio at $v$ over the $l$-th channel. Each of these replications is referred to as a replicated link of $a$. We use $\{a\}^{\tau, \lambda}$ to denote the set of replicated links of $a$, and in general, for each $B \subseteq A$, we use $B^{\tau, \lambda}$ to denote the set of all replicated links of the links in $B$. Clearly, a set of replicated links can transmit successfully at the same time if and only if 1) they are pairwise radio-disjoint, and 2) for each channel $l$, all those replicated links transmitting over channel $l$ are replicated from an independent set of $A$. Any such set is also referred to as an independent set of replicated links. Let $\mathcal{I}^{\tau, \lambda}$ denote the collection of the independent sets of replicated links.

Suppose that $d \in \mathbb{R}_{+}^{A}$ is a link-demand function in terms of transmission time. A link schedule of $d$ is a set

$$
\mathcal{S}=\left\{\left(I_{j}, x_{j}\right) \in \mathcal{I}^{\tau, \lambda} \times \mathbb{R}^{+}: 1 \leqslant j \leqslant q\right\}
$$

satisfying that for each link $a \in A$ :

$$
d(a)=\sum_{j=1}^{q} x_{j}\left|I_{j} \cap\{a\}^{\tau, \lambda}\right|
$$

the two values, $q$ and $\sum_{j=1}^{q} x_{j}$, are referred to as the size and length (or latency) of $\mathcal{S}$ respectively. The minimum length of all link schedules of $d$ is denoted by $\chi^{*}(d)$. The problem of finding a shortest link schedule of $d$ is referred to as Shortest Link Schedule (SLS). The capacity region $\Omega$ of the network consists of all $d \in \mathbb{R}_{+}^{A}$ which has a link schedule of length at most one. In general, the membership test of the capacity region $\Omega$ is NP-complete. A subregion $\Phi$ of $\Omega$ is called a polynomial $\mu$-approximate capacity subregion for some $\mu \geqslant 1$ if it satisfies the following three conditions: 1) $\Phi$ has an explicit representation by a polynomial number of linear inequalities or equalities, 2) there is a polynomial algorithm which produces a link schedule of length at most one for any $d \in \Phi$, and 3) $\Phi$ is a $\mu$-approximation of $\Omega$, i.e., $\Omega \subseteq \mu \Phi$. The first condition ensures the membership of $\Phi$ is polynomial, the second condition implies that $\Phi \subseteq \Omega$, and the third condition ensures that $\Phi$ is "close" to $\Omega$. 
Consider $k$ unicast requests in this wireless network specified by source-destination pairs. For each $1 \leqslant j \leqslant k, \mathcal{F}_{j}$ denotes the set of flows of the request $j$, and the value of a flow $f_{j} \in \mathcal{F}_{j}$ is denoted by $\operatorname{val}\left(f_{j}\right)$. A multiflow is a sequence of flows $f=\left(f_{1}, f_{2}, \ldots, f_{k}\right)$ with $f_{j} \in \mathcal{F}_{j}$ for each $1 \leqslant j \leqslant k$. The cumulative flow of a multiflow $f=\left(f_{1}, f_{2}, \ldots, f_{k}\right)$ is $\sum_{j=1}^{k} f_{j}$. Among all variants of the multiflow problems, the following two are the most basic ones.

- Maximum Weighted Multiflow $(M W M F)$. Given that each request $j$ has a weight $w_{j}$ per unit of its flow, compute a multiflow $f=\left(f_{1}, \ldots, f_{k}\right)$ and a MAC-layer link schedule $\mathcal{S}$ of $\sum_{j=1}^{k} f_{j}$ such that the length of $\mathcal{S}$ is at most one and the total weight of $f$ given by

$$
\sum_{j=1}^{k} \operatorname{val}\left(f_{j}\right) w_{j}
$$

is maximized.

- Maximum Concurrent Multiflow (MCMF). Given that each request $j$ has a traffic demand $d_{j}$, compute a multiflow $f=\left(f_{1}, \ldots, f_{k}\right)$ and a MAC-layer link schedule $\mathcal{S}$ of $\sum_{j=1}^{k} f_{j}$ such that the length of $\mathcal{S}$ is at most one and the concurrency of $f$ given by

$$
\min _{1 \leqslant j \leqslant k} \operatorname{val}\left(f_{j}\right) / d_{j}
$$

is maximized.

The above three problems are closely related to the following two MAC-layer problems.

- Maximum Weighted Independent Set (MWIS). Given a nonnegative weight function $w$ on $A^{\tau, \lambda}$, compute an independent subset $I$ of $A^{\tau, \lambda}$ with maximum total weight $\sum_{e \in I} w(e)$.

- Maximum Independent Set of Links (MISL). Given a subset $B$ of links, compute an independent subset $I$ of $B^{\tau, \lambda}$ with maximum size.

Consider a subset $B$ of $A$. An independent set $I$ of $B^{\tau, \lambda}$ is said to be a maximal independent set (MIS) of $B^{\tau, \lambda}$ if for any link $e \in B^{\tau, \lambda} \backslash I, I \cup\{e\} \notin \mathcal{I}^{\tau, \lambda}$.

\section{Maximizing Wireless Network Capacity Under Protocol Interference Model}

Under the protocol interference model, maximizing wireless network capacity allows for graph-theoretic treatment. The pairwise conflict relations among all node-level links in $A$ under the protocol interference model can be conveniently represented by a link conflict graph $G$. The vertex set of $G$ is $A$ and two links in $A$ are adjacent in $G$ if and only if they conflict with each other. We first introduce two key structural parameters of the protocol interference model, which play critical roles in the algorithm design and analysis.

In our early studies ${ }^{[1-2]}$, we have introduced two types of local independence numbers. For any $a \in A$,
$N[a]$ denotes the set of links in $A$ conflicting with $a$ plus $a$ itself. An orientation of $G$ is a digraph $D$ obtained from $G$ by imposing an orientation on each edge of $G$. Suppose that $D$ is an orientation. For each $a \in A, N_{D}^{\text {in }}[a]$ (respectively, $N_{D}^{\text {out }}[a]$ ) denotes the set of in-neighbors (respectively, out-neighbors) of $a$ in $D$ plus $a$ itself. The inward local independence number (ILIN) of $D$ is defined to be

$$
\max _{a \in A} \max _{I \in \mathcal{I}}\left|I \cap N_{D}^{\mathrm{in}}[a]\right| .
$$

Suppose that $\prec$ is an ordering of $A$. For each $a \in A$, $N^{\prec}[a]$ denotes the set of preceding neighbors of $a$ in $\prec$ plus $a$ itself. The backward local independence number (BLIN) of $\prec$ is defined to be

$$
\max _{a \in A} \max _{I \in \mathcal{I}}\left|I \cap N^{\prec}[a]\right| .
$$

Note that any ordering of $A$ can be treated as an acyclic orientation of $G$. In [1-2], we have discovered orientations (respectively, orderings) with constant ILIN (respectively, BLIN).

In [3], we introduced the interference factor $\rho(a, b)$ of two conflicting node-level links $a$ and $b$, which captures the essential advantage of multiple radios and multiple channels. If $a$ and $b$ share a common endpoint $u$, then each replicated link of $b$ conflicts with exactly

$$
\rho(a, b)=1-\left(1-\frac{1}{\tau(u)}\right)\left(1-\frac{1}{\lambda}\right)
$$

portion of replicated links of $a$; otherwise, each replicated link of $b$ conflicts with exactly

$$
\rho(a, b)=1 / \lambda
$$

portion of replicated links of $a$. In addition, each replicated link of a link $a=(u, v)$ conflicts with exactly

$$
\rho(a, b)=1-\left(1-\frac{1}{\tau(u)}\right)\left(1-\frac{1}{\tau(v)}\right)\left(1-\frac{1}{\lambda}\right)
$$

portion of replicated links of $a$ (include itself). Note that $\rho(a, b)=\rho(b, a)$ in either case.

Subsequently, we present the design and analysis of algorithms for the maximization of the network capacity under the protocol interference model.

\subsection{Maximal Independent Set}

Consider a subset $B$ of $A$. An independent set $I$ of $B^{\tau, \lambda}$ is said to be a maximal independent set of $B^{\tau, \lambda}$ if for any link $e \in B^{\tau, \lambda} \backslash I, I \cup\{e\} \notin \mathcal{I}^{\tau, \lambda}$. Given an ordering $\prec$ of $A$, an MIS $I$ of $B^{\tau, \lambda}$ can be computed greedily as follows. Initialize $I$ to be the empty set, and repeat the following iterations for each link $a=(u, v) \in B$ in the ordering $\prec$. Let $R(u)$ (respectively, $R(v)$ ) be the list of radios of $u$ (respectively, $v$ ) not used by anyone in 
$I$, and let $C(a)$ be the list of channels not used by any preceding links which have conflict with $a$. Compute

$$
k=\min \{|R(u)|,|R(v)|,|C(a)|\} .
$$

If $k>0$, then $I$ is augmented with $k$ replicated links of $a$ using the first $k$ radios from $R(u)$, the first $k$ radios from $R(v)$, and the first $k$ channels from $C(a)$ respectively. Finally, the set $I$ is returned. Clearly, the returned $I$ is a maximal independent set (IS) of $B^{\tau, \lambda}$, and is referred to as the greedy IS of $B^{\tau, \lambda}$ in the ordering $\prec$. In [3], we gave an implementation of the above algorithm whose running time grows linearly with $\lambda$ and $\max _{v \in V} \tau(v)$. In addition, we have proved the following essential property of the greedy IS $I$ of $B^{\tau, \lambda}$ : for any $a \in B$ :

$$
\sum_{b \in N \prec[a]} \rho(a, b)\left|I \cap\{b\}^{\tau, \lambda}\right| \geqslant 1 .
$$

\subsection{Shortest Link Schedule}

Suppose that $d \in \mathbb{R}_{+}^{A}$ is a link-demand function in terms of transmission time. Given a link ordering $\prec$, a greedy scheduling algorithm developed by us in [3] computes a link $\mathcal{S}$ of $d$ iteratively as follows. Initialize $\mathcal{S}$ to the empty set and repeat the following iteration until $d=0$. Let $B$ be the subset of links $a \in A$ with $d(a)>0$. We compute a greedy IS $I$ of $B^{\tau, \lambda}$ in the ordering $\prec$. Let

$$
x=\min _{a \in B} \frac{d(a)}{\mid I \cap\{a\}^{\tau, \lambda \mid},}
$$

and add $(I, x)$ to $\mathcal{S}$. For each $a \in B$, replace $d(a)$ by $d(a)-x\left|I \cap\{a\}^{\tau, \lambda}\right|$. When $d=0$, the algorithm outputs $\mathcal{S}$. The output $\mathcal{S}$ is referred to as the greedy link schedule of $d$ in $\prec$. Clearly, the algorithm has at most $m=|A|$ iterations. The length of the output $\mathcal{S}$ was shown in [3] to be at most

$$
\Delta^{\prec}(d)=\max _{a \in A} \sum_{b \in N^{\prec}[a]} \rho(a, b) d(b) .
$$

Let $\Delta^{*}(d)$ be the minimum value of $\Delta^{\prec}(d)$ among all possible link orders $\prec$. In [3], we show that $\Delta^{*}(d)$ can be achieved by a smallest-last ordering with respect to $d$ which can be computed successively in polynomial time as follows. Initialize $B$ to $A$ and a stack $S$ to be empty. While $B$ is non-empty, choose a link $a \in B$ minimizing

$$
\sum_{b \in N[a] \cap B} \rho(a, b) d(b),
$$

remove it from $B$ and push it onto the stack $S$. When $B$ is empty, $S$ is $A$ and the top-down order of $S$ is a smallest-last ordering of $A$ with respect to $d$.

Now, we are ready to describe our approximation algorithm GreedyLS for $S L S$ developed in [3]. Let $d \in \mathbb{R}_{+}^{A}$ be the input link demand function. The algorithm consists of two steps:

- Step 1: compute the smallest-last ordering $\prec$ with respect to $d$.

- Step 2: compute a greedy link schedule of $d$ in $\prec$. The link schedule $\mathcal{S}$ is referred to as the greedy link schedule of $d$. Its length is at most $\Delta^{*}(d)$.

Next, we present the approximation bound of the greedy link schedule $\mathcal{S}$ of $d$ derived in [3]. Consider an orientation $D$ of $G$ with ILIN $\mu$. For each link $a$, we denote

$$
\Delta_{D}^{\mathrm{in}}(a ; d)=\sum_{b \in N_{D}^{\mathrm{in}}[a]} \rho(a, b) d(b) .
$$

Let

$$
\Delta_{D}^{\mathrm{in}}(d)=\max _{a \in A} \Delta_{D}^{\mathrm{in}}(a ; d) .
$$

Then, if $D$ is acyclic,

$$
\Delta^{*}(d) \leqslant \Delta_{D}^{\mathrm{in}}(d)
$$

otherwise,

$$
\Delta^{*}(d) \leqslant 2 \Delta_{D}^{\mathrm{in}}(d)
$$

on the other hand,

$$
\chi^{*}(d) \geqslant \frac{\Delta_{D}^{\mathrm{in}}(d)}{\mu+2} .
$$

Thus, $\mathcal{S}$ has the approximation bound:

- If there is orientation $D$ with ILIN $\mu$, then the approximation bound of $\mathcal{S}$ is $\mu+2$;

- If there is acyclic orientation $D$ with ILIN $\mu$, then the approximation bound of $\mathcal{S}$ is $2(\mu+2)$.

The orientations given in [1-2] imply the following specific approximation bounds:

- $2\left(\left\lceil\pi / \operatorname{arc} \sin \frac{c-1}{2 c}\right\rceil+1\right)$ in the unidirectional mode in which the interference radius of each link is at least $c$ times the link length for some $c>1$,

- 20 in the bidirectional mode,

- 10 in the bidirectional mode with symmetric interference radii,

- 8 in the bidirectional mode with uniform interference radii.

\subsection{Polynomial Approximate Capacity Subregions}

In [3], we defined a polynomial approximate capacity subregion with respect to an orientation. Consider an orientation $D$ of $G$ with ILIN $\mu$. If $D$ is acyclic,

$$
\Phi_{D}=\left\{d \in \mathbb{R}_{+}^{A}: \Delta_{D}^{\text {in }}(d) \leqslant 1\right\} ;
$$

otherwise, define

$$
\Phi_{D}=\left\{d \in \mathbb{R}_{+}^{A}: \Delta_{D}^{\mathrm{in}}(d) \leqslant 1 / 2\right\} .
$$


The set $\Phi_{D}$ is referred to as the inward capacity subregion with respect to $D$. Then, if $D$ is acyclic, $\Phi_{D}$ is a polynomial $(\mu+2)$-approximation capacity subregion; otherwise $\Phi_{D}$ is a polynomial $2(\mu+2)$-approximation capacity subregion. By adopting any orientation $D$ given in [1-2], the inward capacity subregion with respect to $D$ has the following polynomial approximation bounds:

- $2\left(\left\lceil\pi / \operatorname{arc} \sin \frac{c-1}{2 c}\right\rceil+1\right)$ in the unidirectional mode in which the interference radius of each link is at least $c$ times the link length for some $c>1$,

- 20 in the bidirectional mode,

- 10 in the bidirectional mode with symmetric interference radii,

- 8 in the bidirectional mode with uniform interference radii.

\subsection{Maximum Multiflows}

In [3], we developed a general linear programming (LP) based method for maximum multiflow. We fix an orientation $D$ of the link conflict graph, which could be any one given in [1-2], and use $\mu$ to denote its ILIN. A multiflow $\left(f_{1}, f_{2}, \ldots, f_{k}\right)$ is said to be $\Phi_{D}$-restricted if $\sum_{j=1}^{k} f_{j} \in \Phi_{D}$. A maximum weighted $\Phi_{D^{-r e s t r i c t e d}}$ multiflow is any solution to the following LP of polynomial size

$$
\begin{aligned}
\max & \sum_{j=1}^{k} \operatorname{val}\left(f_{j}\right) w_{j} \\
\text { s.t. } & f_{j} \in \mathcal{F}_{j}, \forall 1 \leqslant j \leqslant k ; \\
& \sum_{j=1}^{k} f_{j} \in \Phi_{D} .
\end{aligned}
$$

Similarly, the maximum concurrent $\Phi_{D}$-restricted multiflow is any solution to the following LP of polynomial size:

$$
\begin{aligned}
\max & \phi \\
\text { s.t. } & f_{j} \in \mathcal{F}_{j}, \forall 1 \leqslant j \leqslant k ; \\
& \operatorname{val}\left(f_{j}\right) \geqslant \phi d_{j}, \forall 1 \leqslant j \leqslant k ; \\
& \sum_{j=1}^{k} f_{j} \in \Phi_{D} .
\end{aligned}
$$

Thus, a maximum weighted (respectively, concurrent) $\Phi_{D}$-restricted multiflow can be computed in polynomial time by linear programming methods. The LP-based method for all multiflow problems in wireless networks runs in three phases.

- Restricted Multiflow Phase. This phase computes maximum weighted (respectively, concurrent) $\Phi_{D}$-restricted multiflow $f=\left(f_{1}, \ldots, f_{k}\right)$.

- Link-Scheduling Phase. This phase computes a greedy link schedule $\mathcal{S}$ of $\sum_{j=1}^{k} f_{j}$.

- Scaling Phase. This phase scales both $f$ and $\mathcal{S}$ down by a factor of the length of $\mathcal{S}$ and then returns them as the final output.
In [3], we proved the LP-based method has an approximation bound $\mu+2$ if $D$ is acyclic, and $2(\mu+2)$ otherwise. By adopting any orientation $D$ given in [1-2], the LP-based method can achieve the following approximation bounds:

- $2\left(\left\lceil\pi / \operatorname{arc} \sin \frac{c-1}{2 c}\right\rceil+1\right)$ in the unidirectional mode in which the interference radius of each link is at least $c$ times the link length for some $c>1$,

- 20 in the bidirectional mode,

- 10 in the bidirectional mode with symmetric interference radii,

- 8 in the bidirectional mode with uniform interference radii.

The LP-based method becomes inefficient in running time and memory when the network size grows. In our most recent work ${ }^{[4]}$, we developed a flow augmentation method which shifts away from the prevailing but inefficient LP-based paradigm for the first time ever. Given an arbitrarily small but positive parameter $\varepsilon$ quantizing the trade-off between the accuracy in terms of the approximation bound and the efficiency in terms of the running time, the flow augmentation method would make a number of shortest-path computations which only grow with $1 / \varepsilon$ in the square order, but still achieve approximation bounds no more than $1+\varepsilon$ times of those achieved with the LP-based method. Such a new method is not only conceptually simpler but also faster. Furthermore, the simplicity of this method allows for easier distributed implementations.

The flow augmentation method replaces the restricted multiflow phase of the LP-based method by a flow augmentation phase. This phase computes a multiflow $f=\left(f_{1}, \ldots, f_{k}\right)$ and its cumulative flow $\sum_{j=1}^{k} f_{j}$ from scratch with successive flow augmentation. Each augmenting flow is transported along the shortest paths of some requests. Despite of the conceptual simplicity, the flow augmentation phase has to address the following technical issues for an algorithmic implementation with targeted accuracy and efficiency:

1) How to quantize the interference-aware link "congestions" and "lengths" with respect to a multiflow?

2) Which shortest path(s) among the $k$ shortest paths should be selected?

3) How much flow should be routed along those selected shortest paths?

4) When should the augmentations terminate? These questions are addressed below.

\subsubsection{Interference-Aware Link Congestions and Lengths}

In wired networks, the congestion of a link due to a multiflow is simply the total amount of flow through this link, and the length of a link capturing the edge 
congestion is simply an exponential function of the congestion of this link only. Such perception on link congestion and length has to be fundamentally changed in wireless networks due to the presence of wireless interference. Intuitively speaking, the "congestion" of a link $a$ due to a multiflow should count both the flow amount through the link $a$ itself and the flows through the links interfering the link $a$. Similarly, the "length" of a link $a$ with respect to a multiflow should take into account both the flow amount through the link $a$ itself and the flows through the links interfered by the link $a$. Subsequently, we will give precise quantizations of such interference-aware link congestions and lengths with respect to a multiflow $f=\left(f_{1}, \ldots, f_{k}\right)$.

The interference-aware congestion of a link $a$ due to $f$, denoted by $\operatorname{cong}_{f}(a)$, is defined to be $\Delta_{D}^{\mathrm{in}}\left(a ; \sum_{j=1}^{k} f_{j}\right)$. The (interference-aware) bottleneck congestion of $f$ is the maximum congestion of $f$ on all links $\Delta_{D}^{\mathrm{in}}\left(\sum_{j=1}^{k} f_{j}\right)$. The interference-aware cost of a link $a$ due to $f$ is defined to be

$$
\operatorname{cost}_{f}(a)=(1+\varepsilon)^{\Delta_{D}^{\mathrm{in}}\left(a ; \sum_{j=1}^{k} f_{j}\right)} .
$$

Such exponential growth of cost in congestion ensures that a highly congested link is less likely to appear in the future augmenting flow. The total interferenceaware cost of $f$ is defined to be

$$
\operatorname{cost}_{f}(A)=\sum_{a \in A} \operatorname{cost}_{f}(a) .
$$

The interference-aware length of a link $a$ with respect to $f$ is defined to be

$$
\ell_{f}(a)=\sum_{b \in N_{D}^{\text {out }[a]}} \rho(a, b) \operatorname{cost}_{f}(a)
$$

For each $1 \leqslant j \leqslant k$, let $\operatorname{dist}_{j}\left(\ell_{f}\right)$ be the length of the shortest path of the request $j$ with respect to $\ell_{f}$. These concepts enable us to establish the following approximate duality between the interference-aware shortest paths and the maximum weighted (respectively, concurrent) multiflows.

- The total weight of the maximum weighted multiflow is at most

$$
\widehat{\mu} \frac{\operatorname{cost}_{f}(A)}{\min _{1 \leqslant j \leqslant k} \operatorname{dist}_{j}\left(\ell_{f}\right) / w_{j}} .
$$

- The concurrency of the maximum concurrent multiflow is at most

$$
\widehat{\mu} \frac{\operatorname{cost}_{f}(A)}{\sum_{j=1}^{k} d_{j} d i s t_{j}\left(\ell_{f}\right)} .
$$

\subsubsection{Flow Augmentation}

For the problem MWMF, each iteration of the flow augmentation phase first computes a shortest path $P_{j}$ of each request $j$ with respect to the interference-aware length function $\ell_{f}$ and then computes the price $\frac{\ell_{f}\left(P_{j}\right)}{w_{j}}$ of each request $j$. For the least-priced request $j$, the augmenting flow would route a flow of value

$$
\delta=\frac{1}{\max _{a \in A} \rho\left(N_{D}^{\mathrm{in}}[a] \cap P_{j}, a\right)},
$$

along $P_{j}$, and $f_{j}$ is updated accordingly. The above value of $\delta$ is adopted to ensure that the bottleneck interference-aware congestion of this augmenting flow is exactly one.

For the problem MCMF, each iteration of the flow augmentation phase first computes a shortest path $P_{j}$ of each request $j$ with respect to the interference-aware length function $\ell_{f}$ and then computes a concurrency

$$
\sigma=\frac{1}{\max _{a \in A} \sum_{j=1}^{k} d_{j} \rho\left(N_{D}^{\mathrm{in}}[a] \cap P_{j}, a\right)} .
$$

For each $1 \leqslant j \leqslant k$, the augmenting multiflow would route a flow of value $\sigma d_{j}$ along $P_{j}$, and the flow $f_{j}$ is updated accordingly. The above value of $\sigma$ is selected such that the bottleneck interference-aware congestion of this augmenting multiflow is exactly one.

The above iterations are repeated for

$$
\left\lceil\frac{m \ln m}{\ln (1+\varepsilon)-\frac{\varepsilon}{1+\varepsilon}}\right\rceil
$$

times. The running time of the flow augmentation phase increases with $1 / \varepsilon$ in at most the square order and does not depend on the number of channels and the number of radios at each node. Within each iteration of the flow augmentation phase, the computation of the $k$ shortest paths can be accelerated by exploiting the capability of the Dijkstra's shortest-path algorithm that may compute the shortest path from a common source node to all other nodes. Therefore, we group the requests by a common source node, and the number of groups is at most $\min \{n, k\}$. The shortest paths of all requests in the same group can be computed by a single call of the Dijkstra's shortest-path algorithm. For $k=\Theta\left(n^{2}\right)$, this implementation is a linear factor speedup over the naive implementation. The final output was proved in [4] to have an approximate bound $(1+\varepsilon)(\mu+2)$ if $D$ is acyclic and $2(1+\varepsilon)(\mu+2)$ otherwise.

\section{Maximizing Wireless Network Capacity Under Physical Interference Model}

Maximizing the wireless network capacity under physical interference model is notoriously hard due to the non-locality and the additive nature of the wireless interference under the physical interference model. The graph-theoretic approach followed by the maximization 
of the wireless network capacity under protocol interference model can no longer be applied to the maximization of the wireless network capacity under physical interference model. Our initial algorithmic studies on maximizing the wireless network capacity under physical interference model took a different technical approach. The following algorithmic reductions were established in [5] in SC-SR setting but can be extended to MC-MR setting as well.

- If MWIS has a polynomial $\mu$-approximation algorithm, then all of MWMF, MCMF and SLS have a polynomial $\mu$-approximation algorithm.

- If MIS has a polynomial $\mu$-approximation algorithm, then all of MWIS, MWMF, MCMF and SLS have a polynomial $O(\ln \alpha) \mu$-approximation algorithm, where $\alpha$ is the size of largest independent set.

In addition, we have established the following general algorithmic reduction recently. If MWIS has a polynomial $\mu$-approximation algorithm in SCSR setting, then MWIS has a polynomial $(\mu+2)$ approximation algorithm in MC-MR setting. By combining these algorithmic reductions, we obtain the following general algorithmic reductions.

- If MWIS in SC-SR setting has a polynomial $\mu$ approximation algorithm, then all of MWMF, MCMF and SLS in MC-MR setting have a polynomial $(\mu+2)$ approximation algorithm.

- If MIS in SC-SR setting has a polynomial $\mu$ approximation algorithm, then all of MWIS, MWMF, MCMF and SLS in MC-MR setting have a polynomial $O(\ln \alpha)(\mu+2)$-approximation algorithm, where $\alpha$ is the size of largest independent set.

These algorithmic reductions are leveraged by our studies $^{[6-8]}$ as described below, depending on whether power control is required or not.

No Power Control. In the setting of no power control, an assignment of transmission power to links is pre-specified, and a set $I$ of links is independent if and only if all links in $I$ can communicate successfully at the same time under the physical interference model. A power assignment is said to be monotone if the transmission power of a link is non-decreasing with the link length, and to be sub-linear if the received power by a link is non-increasing with the link length. Two extreme and common monotone power assignments are the uniform power assignment and the linear power assignment. A uniform power assignment assigns the same transmission power to all links; a linear power assignment ensures that all links have the same received power. With a monotone and sublinear assignment, we have explored the rich natures of the physical interference and exploited them to develop approximation algorithms for MIS in both the unidirectional mode ${ }^{[7]}$ and the bidirectional mode ${ }^{[6]}$. Our algorithms not only output an independent set with stronger properties but also have a much significantly smaller constant approximation bound. Our better approximation bounds also immediately lead to improved logarithmic approximations for other wireless link scheduling problems including SLS, MWIS, MWMF, and MCMF. With linear power assignment, we improve the status quo. By exploring and exploiting the rich nature of the wireless interference with the linear power assignment, we developed the first and only constant-approximation algorithms for MWIS, SLS, MWMF, and MCMF in [9].

Power Control. In the setting of power control, a set $I$ of links is independent if and only if there exists a transmission power assignment to $I$ at which all links can communicate successfully at the same time under the physical interference model. The problem MIS with power control involves the joint selection and power assignment of a largest number of given links which can communicate successfully at the same time under the physical interference model. All prior studies on MIS with power control in either bidirectional mode or unidirectional mode have to assume unbounded maximum transmission power for the purpose of avoiding the technical obstacle due to the ambient noise. The algorithms developed by us in [6] and [8] not only outperform existing work, but also are applicable to bounded maximum transmission power, arbitrary path-loss exponent and arbitrary dimension of the deployment space. Both the design and the analysis of our approximation algorithms are built upon the new power assignments including canonical mean power assignment and canonical iterative power assignments developed by our studies. Our algorithms not only output an independent set with stronger properties but also have a much significantly smaller constant approximation bound. These algorithms immediately lead to logarithmic approximations for MWIS, SLS, MWMF, and MCMF.

\section{Maximum Stable-Efficiency Link Scheduling}

In [10-11], we conducted a queuing-theoretic study of wireless link scheduling in MC-MR wireless networks under the protocol interference model. A wireless link scheduling algorithm takes as an input a set of nodelevel links together with their backlogs of packets waiting for transmission, and outputs a set of packets which can be transmitted successfully at the same time. We assume that time is slotted and for each $t \in \mathbb{N}$, the $t$ th time slot is the time interval $(t-1, t]$. Any packet arriving in a slot is assumed to arrive at the end of the slot, and may only be transmitted in the subsequent slots. In addition, the packet arrivals are assumed to 
be mutually independent and temporally independent and identical processes with arrival rate vector $\boldsymbol{d}$. Consider a wireless link scheduling algorithm $\mathcal{A}$. In each time-slot, a set of backlogged packets are selected to be transmitted using the algorithm $\mathcal{A}$. Let $\boldsymbol{X}(t)$ (respectively, $\boldsymbol{Y}(t))$ denote the vector of the cumulative number of packets arriving (respectively, transmitted) in the first $t$ time slots, and $\boldsymbol{Z}(t)$ denote the vector of the number of packets queued at the very end of time slot $t$. Then,

$$
\boldsymbol{Z}(t)=\boldsymbol{Z}(0)+\boldsymbol{X}(t)-\boldsymbol{Y}(t) .
$$

The network is said to be stable if the Markov chain $(\boldsymbol{Z}(t))$ is positive recurrent. The stability region of the wireless link scheduling algorithm $\mathcal{A}$, denoted by $\Lambda$, is the set of arrival rate vectors $\boldsymbol{d}$ such that the network is stable. Since the maximum stability region of the network, which consists of arrival rate vectors such that there exists a scheduling policy stabilizing the network, is the set

$$
\Omega^{o}=\left\{\boldsymbol{d} \in \mathbb{R}_{+}^{A}: \chi^{*}(\boldsymbol{d})<1\right\},
$$

the stable efficiency of the wireless link scheduling algorithm $\mathcal{A}$ is defined to be

$$
\sup \left\{\gamma \in \mathbb{R}_{+}: \Lambda \supseteq \gamma \Omega^{o}\right\} .
$$

The problem Maximum Stable-Efficiency Link Scheduling seeks a wireless link scheduling algorithm with the largest stable efficiency.

Given a link ordering $\prec$, a greedy link scheduling algorithm (GLS) developed by us in [3] schedules the transmissions by all links in $\prec$ as follows. Each link transmits as many packets as possible from its queue using the radios at its two endpoints which have not been used by any preceding links and the channels which have not been used by any preceding conflicting links. The choice of $\prec$ is essential to both the implementation simplicity and the throughput efficiency. A link ordering $\prec$ is dynamic if it may vary with the queue lengths of the links, and static if it does not vary with the queue lengths of the links. The GLS in a dynamic link ordering requires the expensive recomputation of the link ordering in each time-slot, and, in return, it may achieve better stable efficiency. On the other hand, the GLS in a static link ordering only needs a single precomputation of the link ordering and therefore enjoys much simpler implementation.

A renowned dynamic link ordering is the LongestQueue-First (LQF) ordering, which sorts the links in the decreasing order of backlog lengths. In [10], we derived a stability subregion of the GLS in LQF ordering with closed form

$$
Q^{*}=\left\{\boldsymbol{d} \in \mathbb{R}_{+}^{A}: \Delta^{*}(\boldsymbol{d})<1\right\},
$$

and proved that $\gamma \Omega^{\circ} \subseteq Q^{*}$ where $\gamma$ is

- $1 /\left(2\left(\left\lceil\pi / \operatorname{arc} \sin \frac{c-1}{2 c}\right\rceil+1\right)\right)$ in the unidirectional mode,

- $1 / 20$ in the bidirectional mode,

- $1 / 10$ in the bidirectional mode with symmetric interference radii,

- $1 / 8$ in the bidirectional mode with uniform interference radii.

The value of $\gamma$ is thus a lower bound on the throughput efficiency ratio of the LQF scheduling.

For an arbitrary static link ordering $\prec$, we derived in [11] a stability subregion of the static GLS in $\prec$ with closed form

$$
Q^{\prec}=\left\{\boldsymbol{d} \in \mathbb{R}_{+}^{A}: \Delta^{\prec}(\boldsymbol{d})<1\right\},
$$

and proved that

$$
\frac{1}{\mu+2} \Omega^{o} \subseteq Q^{*}
$$

where $\mu$ is the BLIN of $\prec$. Hence, the stable efficiency ratio of the GLS in $\prec$ is at least $\frac{1}{\mu+2}$. Motivated by this discovery, we adopt one of the following two static orderings in the bidirectional mode.

- Interference Radius Decreasing Ordering. The interference radius of a link is defined to be the larger one of the interference radii of its endpoints, and all links are sorted in descending order of the interference radius.

- Lexicographic Ordering. All links are sorted in the lexicographic order of their left endpoints.

With arbitrary interference radii, the GLS in interference radius decreasing ordering has stable efficiency at least $1 / 25$. With symmetric interference radii, the GLS in interference radius decreasing ordering has stable efficiency at least $1 / 10$. With uniform interference radii, the GLS in lexicographic ordering has stable efficiency at least $1 / 8$.

The membership of both $Q^{*}$ and $Q^{\prec}$ can be tested in polynomial time. This computational tractability is particularly favorable for cross-layer optimization, where one needs to allocate the link rates efficiently while still ensuring the network stability. In contrast, such stability subregions of computational tractability were even missing in SC-SR wireless networks prior to our studies. Since $Q^{\prec} \subseteq Q^{*}$, the stability subregion $Q^{\prec}$ of the GLS in $\prec$ is also a stability subregion of the GLS in LQF ordering. On the other hand, with uniform (respectively, symmetric) interference radii, both the GLS in LQF ordering and the GLS in lexicographic ordering (respectively, interference radius decreasing ordering) have the same lower bounds on the stable efficiency. With arbitrary interference radii, the GLS in LQF ordering only has slightly better stable efficiency than the 
GLS in interference radius decreasing ordering. Therefore, with uniform (respectively, arbitrary) interference radii, we advocate the GLS in lexicographic ordering (respectively, interference radius decreasing ordering) due to its implementation simplicity and comparable stable efficiency.

All the existing studies on the stability analysis of a greedy link scheduling in SC-SR wireless networks established the stability by a classic fluid-limit criterion in the general context of multiclass queuing networks. One crucial condition of this criterion is that the queuing service discipline is working-conserving: a server is idle only when there is no customer waiting for the service. Apparently, the greedy scheduling in wireless networks is not working-conserving, as a link with non-empty queue/backlog may be idle due to the interference from other nearby links. So, the direct applicability of classic fluid-limit criteria to wireless link scheduling is questionable. Instead, our stability analyses were accomplished by an innovative sample-path argument, which pushes the deterministic arguments as far as possible while trying to avoid the heavy machinery of stochastic processes. A clear advantage of this approach is that the algorithmic-theoretic discoveries can be easily leveraged to establish the stability. Sample-path argument also helps pinpoint what and when stochastic conditions are needed to guarantee the stability. For example, the packet arrival process in [10-11] is only required to be mutually independent and temporally i.i.d., while the packet arrival processes in all existing work have to meet additional conditions.

\section{Conclusions}

The NSFC Project 61128005 conducted comprehensive algorithmic-theoretic and queuing-theoretic studies of maximizing wireless networking capacity in MCMR wireless networks and fundamentally advanced the state of the art. We expect the new techniques and tools developed in this project will have wide applications in capacity planning, resource allocation and sharing, and protocol design for wireless networks, and will serve as the basis for future algorithm developments in wireless networks with advanced features, such as multi-input multi-output (MIMO) wireless networks.

\section{References}

[1] Wan P. Multiflows in multihop wireless networks. In Proc. the 10th ACM MOBIHOC, May 2009, pp.85-94.
[2] Wan P, Cheng Y, Wang Z, Yao F. Multiflows in multi-channel multi-radio multihop wireless networks. In Proc. the 2011 IEEE INFOCOM, April 2011, pp.846-854.

[3] Wan P, Jia X, Dai G et al. Scalable algorithms for wireless link schedulings in multi-channel multi-radio wireless networks. In Proc. the 2013 IEEE INFOCOM, Apr. 2013, pp.2121-2129.

[4] Wan P, Wang Z, Wang L et al. From least interference-cost paths to maximum (concurrent) multiflow in MC-MR wireless networks. In Proc. the 2014 IEEE INFOCOM, April 2014, pp.334-342.

[5] Wan P, Frieder O, Jia X, Yao F, Xu X H, Tang S. Wireless link scheduling under physical interference model. In Proc. the 2011 IEEE INFOCOM, April 2011, pp.838-845.

[6] Wan P, Chen D, Dai G et al. Maximizing capacity with power control under physical interference model in duplex mode. In Proc. the 2012 IEEE INFOCOM, Mar. 2012, pp.415-423.

[7] Ma C, Al-dhelaan F, Wan P. Maximum independent set of links with a monotone and sublinear power assignment. In Proc. the 8th WASA, Aug. 2013, pp.64-75.

[8] Ma C, Al-dhelaan F, Wan P. Maximum independent set of links with power control. In Proc. the 8th WASA, Aug. 2013, pp.474-485.

[9] Wan P, Wang L, Ma C et al. Maximizing wireless network capacity with linear power: Breaking the logarithmic barrier. In Proc. the 2013 IEEE INFOCOM, April 2013, pp.135-139.

[10] Wan P, Xu X, Wang Z et al. Stability analyses of longestqueue-first link scheduling in MC-MR wireless networks. In Proc. the 13th ACM MOBIHOC, June 2012, pp.45-54.

[11] Wan P, Wan Z, Wang Z et al. Stability analyses of static greedy link schedulings in MC-MR wireless networks. In Proc. the 2013 IEEE INFOCOM, April 2013, pp.2868-2876.

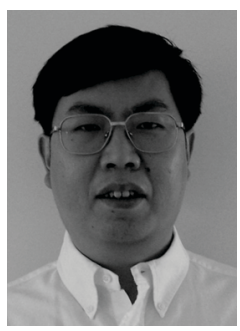

Pengjun Wan is currently a professor of computer science in the Department of Computer Science, Illinois Institute of Technology, Chicago. He received his B.S. degree from Tsinghua University, M.S. degree from the Chinese Academy of Science, and Ph.D. degree in computer science from the University of Minnesota. His research interests include wireless networks, and algorithm design and analysis.

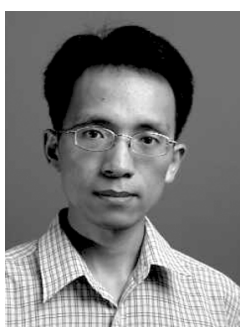

Zhi-Guo Wan is an assistant professor in School of Software, Tsinghua University, Beijing. His main research interests include applied cryptography, system security and privacy protection for various systems. He received his B.S. degree from Tsinghua University in 2002, and Ph.D. degree from School of Computing, National University of Singapore, in 2007, both in computer science. He worked as a postdoctoral researcher in Katholieke University of Leuven, Belgium, during 2006 2008. 